\title{
HER2 gene and protein expression status of breast carcinoma can be reliably tested on a single slide
}

\author{
Marie-Pierre Chenard ${ }^{1}$ • Marie-Pierre Wissler ${ }^{1}$ - Noëlle Weingertner ${ }^{1}$. \\ Carole Mathelin ${ }^{2}$. Jean-Pierre Bellocq ${ }^{1}$
}

Received: 16 September 2013 / Revised: 23 February 2015 / Accepted: 4 May 2015 /Published online: 19 May 2015

(C) The Author(s) 2015. This article is published with open access at Springerlink.com

\begin{abstract}
Human epidermal growth factor receptor 2 (HER2) status in breast carcinomas serves as a predictor of benefit from anti-HER2 therapy. In providing clinicians with the information necessary to decide whether or not to treat with targeted therapy, it might be necessary to choose between methods assessing HER2 protein overexpression or gene amplification. A new diagnostic approach could be a combination of both tests on the same slide. If accurate and reproducible, this approach might optimize patient stratification for therapy. In this study, formalin-fixed paraffin-embedded tumor samples from 77 breast cancer patients were examined for HER2 by immunohistochemistry (IHC) and silver in situ hybridization (SISH) using HER2 IHC (clone 4B5), HER2/CEN17SISH, and combined IHC and SISH assay, called gene protein (GP). Cases were selected to ensure a sufficient number of borderline cases on the basis of IHC scores $(0,1+, 2+, 3+)$, obtained during diagnostic histopathological workup. The concordance between the HER2 IHC score obtained during diagnostic histopathological workup
\end{abstract}

Marie-Pierre Chenard

marie-pierre.chenard@chru-strasbourg.fr

Marie-Pierre Wissler

marie-pierre.wissler@chru-strasbourg.fr

Noëlle Weingertner

noelle.weingertner@chru-strasbourg.fr

Carole Mathelin

carole.mathelin@chru-strasbourg.fr

Jean-Pierre Bellocq

jean-pierre.bellocq@chru-strasbourg.fr

1 Department of Pathology, University Hospitals of Strasbourg, 1 avenue Molière, 67098 Strasbourg, France

2 Department of Gynecology and Obstetrics, University Hospitals of Strasbourg, Strasbourg, France and GP was $93 \%$. Discordances had no influence on therapy decisions. The concordance between ISH results using dual ISH (DISH) and GP was $96 \%$. Of the 77 cases studied by GP, three cases with a ratio close to 2 would have been called amplified by DISH. The use of GP reduced the time for slide reading for a trained pathologist by up to $25 \%$, relative to sequential reading of IHC followed by SISH. For cases with an IHC score of $2+$, the final result was obtained in 1 day, while the sequential technique would have required retesting by ISH on a second day. In conclusion, assessment of HER2 status by GP is an improvement for pathologists and facilitates clinical decision-making for breast cancer management.

Keywords HER2 - Breast carcinomas - 4B5 - SISH · Gene · Protein expression $\cdot$ Simultaneous

\section{Introduction}

Human epidermal growth factor receptor 2 (HER2) is a protein on the surface of cancer cells that stimulates tumor growth. When the HER 2 gene is amplified, it triggers overproduction, commonly called overexpression, of HER2 [1] protein. Tumors that strongly overexpress HER 2 and/or those with a proven amplification of the $H E R 2$ gene are classified as being HER2 positive. HER2-positive cancers are associated with poor overall prognosis with faster time to relapse or progression at all stages [2-4]. In the early days of HER2 testing, amplification of the HER2 gene and the corresponding overexpression of HER 2 protein was found in approximately 25 to $30 \%$ of breast cancer [5], but this rate was probably an overestimate as it is now identified in approximately 15 to $20 \%$ [6] of primary breast cancer cases, while recent data show a further decreasing trend to around $14 \%$ [7]. 
Assessment of HER2 status in the breast is required to support treatment decisions, as it predicts response to HER2 targeted therapies. Presently, four HER2-targeted therapies are approved by the Food and Drug Administration (FDA) for treatment of HER2-positive breast cancer: Trastuzumab ${ }^{\circledR}$, Lapatinib $^{\circledR}$, Pertuzumab ${ }^{\circledR}$, and Trastuzumab emtasine ${ }^{\circledR}$. In addition, promising new approaches are being developed including monoclonal antibodies and small-molecule tyrosine kinase inhibitors targeting HER 2 or other HER family members, antibodies linked to cytotoxic moieties or modified to improve their immunological function, immunostimulatory peptides, and PI3K and IGF-1R pathway $[8,9]$ targets.

To date, only two techniques for HER2 status determination are validated, FDA approved, and broadly used in a diagnostic setting. Immunohistochemical (IHC) analysis identifies HER2 protein expression on the cell surface, while in situ hybridization (ISH) determines the degree of HER2 gene amplification. Both methods are highly specific and reproducible when performed under standardized and validated conditions. HER2 testing has been standardized for breast carcinoma, and the American Society of Clinical Oncology (ASCO) recommends that HER2 status should be determined for all invasive breast cancers [10, 11]. The ASCO/CAP 2007 HER2 guidelines provide an algorithm defining positive and negative status for both HER2 protein expression and gene amplification. Cases demonstrating IHC staining of $3+$ (uniform, complete intense membranous staining of more than $30 \%$ of invasive tumor cells) or an ISH HER 2 copy number $\geq 6$ or a ratio HER2 gene signal to chromosome 17 signal $\geq 2.2$ are considered positive. A negative result is IHC staining of 0 or $1+$ or ISH HER 2 copy number $<4$ or a ratio $<1.8$. The recently revised ASCO-CAP 2003 guidelines state that all tumors with complete, intense circumferential membrane staining of more than $10 \%$ of the cells are considered as $3+$ and therefore positive. HER2 status by ISH is positive for cases showing a HER2 gene to chromosome 17 signal ratio $\geq 2$, regardless of the number of HER 2 copies. Cases with a HER2 gene to chromosome 17 ratio $<2$ but with a HER 2 copy number of 6 or higher are also considered positive.

Cases with complete membrane staining, that is either nonuniform or weak in intensity but with obvious circumferential distribution in at least $10 \%$ of cells (IHC $2+$ ), are considered equivocal and require assessment by ISH [19]. This group accounts for approximately $15 \%$ of all tumors and, for optimal guidance of HER2 targeted therapy, further quantification of gene copy number to determine amplification status is needed [12]. In the revised version of the ASCOCAP guidelines, the definition of IHC equivocal cases changed to tumors with circumferential membrane staining that is incomplete and/or weak/moderate in more than $10 \%$ of tumors cells, or with intense complete circumferential membrane staining in less than or equal to $10 \%$ of the tumor cells.
For cases with an equivocal ISH ratio of 1.8-2.2, additional cells should be scored to allow a diagnostic decision to be reached. A ratio of 1.80-1.99 should be reported as borderline not amplified and HER2 negative and a ratio of 2.00-2.20 as borderline amplified and HER2 positive. It is recommended that laboratories show at least $95 \%$ concordance between IHC and ISH. The revised version of the ASCO-CAP guidelines also changes the definition for equivocal cases defined by ISH testing. Cases with an HER2 gene copy to chromosome 17 signal ratio above 2 or with an HER 2 signal number between 4 and 6 are considered equivocal.

Based on the ASCO guidelines, the currently most widely used testing algorithm for breast carcinoma consists of firstline HER2 IHC staining followed by ISH testing for $2+$ cases. This algorithm may result in some discordant cases, e.g., IHC 0 or $1+$ cases amplified in ISH or IHC $3+$ cases nonamplified in ISH, amounting to about $4 \%$ of cases as described by Lee et al. [13] and Bernasconi et al. [14]. In addition, intratumor heterogeneity of HER2 gene amplification has been observed in breast cancer, ranging from a few [15] to up to $36 \%$ of the amplified cases [16], which introduces additional difficulties in HER2 status evaluation especially for equivocal cases [17]

The long history of HER2 testing in breast cancer is an indication of the importance of continuing efforts to improve HER2 diagnostic assays and their interpretation, to ensure that patients who may benefit receive the appropriate targeted therapy. A new robust and reliable method, that would allow assessment of protein expression and gene amplification status on the same slide, could be a significant improvement. The aim of this study is to test the feasibility and reliability of this new method focusing especially on borderline breast cancer cases.

\section{Materials and methods}

\section{Study design}

A new gene and protein detection platform (GP, Ventana) combines IHC staining with ISH. To estimate HER2 GP reliability, results were compared with FDA-approved INFORM HER2 DISH DNA Probe Cocktail and HER2 IHC (clone 4B5) assays (Ventana).

\section{Patients}

Patients were selected based upon previously performed diagnostic IHC staining, with the intention to include a sufficient number of borderline cases. This resulted in a case series of 10 cases without IHC overexpression (0), 18 with weak staining $(1+), 35$ with moderate staining $(2+)$, and 14 with strong 
staining (3+). For this study, HER2 IHC status of all cases was confirmed by a second IHC analysis done in one staining run.

\section{Immunohistochemical analysis}

Formalin-fixed paraffin-embedded sections were dried at $60{ }^{\circ} \mathrm{C}$ for $2 \mathrm{~h}$ and then processed on a BenchMark XT automated slide-stainer (Ventana). All used reagents were from Ventana. After deparaffinization and pretreatment (Cell Conditioning I for $30 \mathrm{~min}$ at $95^{\circ} \mathrm{C}$ ), sections were incubated with primary antibody (clone 4B5) for 20 min at $37^{\circ} \mathrm{C}$ and secondary antibody (HRP-conjugated goat anti-rabbit antibody). Immunoreactivity was detected with the iView DAB Detection Kit, and counterstaining was performed with Hematoxylin II.

\section{Dual color in situ hybridization}

Formalin-fixed paraffin-embedded sections were dried at $60{ }^{\circ} \mathrm{C}$ for $2 \mathrm{~h}$ and then processed on a BenchMark XT automated slide-stainer (Ventana). All used reagents were from Ventana. After deparaffinization and pretreatment (Cell Conditioning II, protease, denaturation), sections were exposed to probe hybridization (Chromosome 17 centromere labeled with DIG and HER2 labeled with DNP) with HybReady and stringency washing using SSC solution. The DNP probe was detected with ultraView SISH DNP [black dots] and the DIG probe with the ultraView Red [red dots], and the slides were counterstained with hematoxylin.

\section{HER2 gene and protein platform}

Formalin-fixed paraffin-embedded sections were dried at $60{ }^{\circ} \mathrm{C}$ for $2 \mathrm{~h}$ and then processed on a BenchMark XT automated slide-stainer (Ventana). All used reagents were from Ventana. The staining procedure in essence consisted of subsequent application of the IHC and double ISH procedures as described above. HybClear is a unique reagent specific to the Ventana GP platform that enables combination of both techniques on a single slide by blocking silver dust background. The total duration of the protocol was about $15 \mathrm{~h}$.

\section{Scoring of staining results}

IHC and ISH results were scored by an experienced breast pathologist (MPC) only in invasive carcinoma, by bright field microscopy using $\times 20, \times 40$, and $\times 60$ objectives. IHC staining results were classified according to ASCO-CAP 2007 guidelines taking into account staining intensity, localization, and extent ( $\%$ of positive staining cells) into $0,1+, 2+$, and $3+$ groups. ISH signals were reported as single copies, multiple copies, and clusters. Normal HER2 or CEN17 signals (one to two copies/cell in stromal fibroblasts, endothelial cells, lymphocytes, and benign breast epithelial cells) served as internal positive control, which was considered as adequate when the signals were visible in the sample at any magnification used. A minimum of 10 cells were scored in cases without variation between nuclei or clustered signals, and 20 to 40 cells were scored for cases with $2+$ IHC and/or internuclear variation. The number of HER2 signals was divided by the number of CEN17 signals to obtain a HER2/CEN17 ratio.

GP-stained slides were first evaluated at low power to score HER2 expression, as performed for only IHC-stained slides. An ISH score was then established at higher magnification. Slides were read in a randomized manner by session of 45 slides (15 IHC, 15 ISH, and 15 GP slides). To avoid influence of previously seen slides on scoring, within a reading session IHC, ISH, and GP were independently scored and subsequent sessions never concerned the same 15 cases. The duration of scoring was measured for each category of slides using 0.5 min increments.

\section{Results}

\section{Visual appreciation}

Single IHC staining was successful on all cases. Dual SISH was successful initially on 69 cases and with an additional staining run on 3 more cases. GP staining was successful on 74 cases after the first run and on 3 more cases with a second run. This left 72 cases for which results of the 3 staining procedures were available.

The first impression when looking at GP-stained slides was that immunostaining is less crisp, which might be due to the pretreatment required for the DISH step. However, this had no impact on reading or scoring, following ASCO-CAP 2007 or the recently published ASCO-CAP 2013 guidelines, although reading GP-stained slides might require some time for an experienced pathologist to adapt (Fig. 1). The quality of DISH staining obtained with GP was judged as good as the with DISH staining only. In some HER2 3+ cases, DISH staining resulted in smaller dots or clusters, or areas of strong membranous DAB staining without ISH signals. In these cases, IHC $3+$ score and/or an HER2/CEN17 ratio of 2 (or more than five copies of HER2) in other areas allowed valid HER2 status assessment (Fig. 1).

\section{Concordance of IHC on single and GP-stained slides}

The concordance between HER2 expression using IHC only and GP was $93 \%$ (Table 1). Minor discrepancies were observed for $1+$ cases, without any impact on HER2 status assessment. 

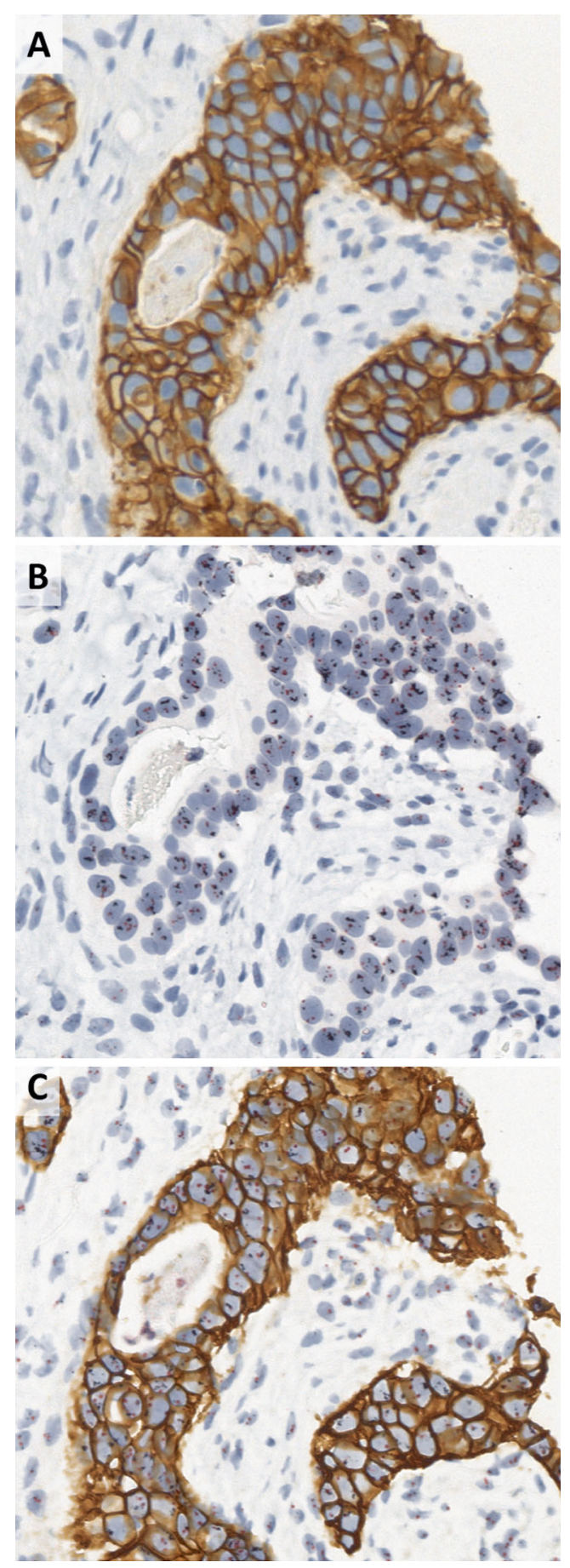

Fig. 1 IHC (a), DISH (b), and GP (c) staining of the same breast carcinoma case at $\times 40$. HER2 IHC score is determined as $3+$ for both IHC and GP, with similar staining pattern and intensity. Similar amplification with clusters is observed in both DISH and GP techniques

\section{Concordance of ISH on DISH and GP-stained slides}

The concordance between the HER2/CEN17 ratios obtained on DISH and GP-stained slides was $94 \%$ (96\% when reduced to amplified or not amplified status, Tables 2 and 3). In three cases, DISH and GP results were discrepant. These were
Table 1 Comparison of HER2 IHC score determined on IHC and GP slides

\begin{tabular}{llllll}
\hline & & \multicolumn{2}{l}{ IHC } & & \\
\cline { 3 - 6 } & & 0 & $1+$ & $2+$ & $3+$ \\
\hline GP & 0 & 7 & 4 & 0 & 0 \\
& $1+$ & 1 & 11 & 0 & 0 \\
& $2+$ & 0 & 0 & 36 & 0 \\
& $3+$ & 0 & 0 & 0 & 13 \\
\hline
\end{tabular}

borderline cases, corresponding to those reported in the literature with interobserver and intraobserver variability (HER2/CEN17 between 1.8 and 2.2).

\section{Concordance of HER2 status on IHC + ISH and GP-stained slides}

Using the previously described algorithm (first HER2 IHC and DISH only for $2+$ cases), with one exception all cases were concordant (Table 4). The discordant case was scored as 0 by IHC in single staining IHC and GP but ISH amplified by both DISH and GP staining (mean HER2 copy number $>6$, HER2/CEN17 <2 due to polysomy) (Fig. 2), even though close to the cutoff value (6.5 for DISH and 6.05 for GP).

\section{Reading time comparing IHC + ISH with GP}

The regression curve comparing reading time to result of IHC followed by DISH with GP showed $y=0.7355$, corresponding to a gain in time of reading of $25 \%$, notably when focusing on borderline (1+ and $2+$ IHC) cases (data not shown).

\section{Discussion}

In this study, we tested a new technique for simultaneous assessment of HER2 protein expression and gene amplification status. This technique was first described by Nitta et al. [18] and Hierschmann et al. [19] and optimizes determination of HER2 status as well as allowing identification of cases in which HER2 IHC and HER2 ISH statuses are not concordant. Although breast cancer heterogeneity has been described as a major cause of variability in the assessment of HER2 status, this was not the focus of our study. The possibility to observe simultaneously HER2 expression and HER2 amplification will guide the observer to the most relevant tumor area and

Table 2 Comparison of HER2 IHC score determined on IHC and GP slides classified as NEG (negative) for 0 and $1+$ and POS (positive) as $2+$ and $3+$ cases

\begin{tabular}{llll}
\hline & & \multicolumn{1}{l}{ IHC } & \\
\cline { 3 - 4 } & & NEG & POS \\
\hline GP & NEG & 23 & 0 \\
& POS & 0 & 49 \\
\hline
\end{tabular}


Table 3 Comparison of HER2 ISH score determined on DISH and GP slides classified as NEG (negative) when case show a ratio HER2/Chromosome 17 below 2 and less than six copies and POS (positive) when the case show a ratio above 2 or a mean copy number of HER2 gene above or equal 6

\begin{tabular}{llll}
\hline & & \multicolumn{2}{c}{ DISH } \\
\cline { 3 - 4 } & & Neg & Pos \\
\hline GP & Neg & 46 & 2 \\
& Pos & 1 & 23 \\
\hline
\end{tabular}

also show areas in which HER2 amplification is not accompanied by protein overexpression. Such differences might be related to preanalytical parameters or to as-yet unidentified biological events [20] .

Although IHC and ISH are robust and reliable techniques, misclassification does occur following the ASCO-CAP testing algorithm [14], which might incur changes in patient treatment. Only patients with a positive HER2 status, either HER2 IHC $3+$ or amplified, will be treated with one of the anti HER2 therapies [23-27]. In our study, one case did not show overexpression (scored as 0 both in IHC only and GP) but was amplified (in both techniques, Fig. 2). This case would have been classified as negative using the ASCO-CAP testing algorithm. However, as the case showed more than six copies of HER2 although it was polysomic (ratio of HER2/Chromosome 17 below 2), following the same ASCO-CAP rules this patient qualifies for treatment with targeted anti-HER2 therapy.

We found the GP assay more robust than the DISH assay. After GP staining, $96 \%$ of the slides could be scored while this was the case for only $90 \%$ of the DISH-stained slides. This may be due to the stronger pretreatment necessary in GP staining as it consists of successive pretreatments for IHC and for DISH (the latter being similar to DISH only but longer and with a higher protease concentration).

In our institution, the workflow for HER2 testing of IHC $2+$ cases requires up to 3 days because of the need for additional ISH testing. This is acceptable but may become problematic when due to technical failure a second staining round would be required especially for ISH, as in such event a final result for HER2 status assessment might take up to 1 week. Using GP, complete HER2 status can be determined in 1 day,

Table 4 Comparison of HER2 status using combination of HER2 IHC and DISH techniques vs. GP

\begin{tabular}{llll}
\hline & & \multicolumn{2}{l}{ IHC + DISH } \\
\cline { 3 - 3 } & & Neg & Pos \\
\hline GP & Neg & 48 & 0 \\
& Pos & 1 & 23 \\
\hline
\end{tabular}

Positive and negative cases are defined according to ASCO-CAP guidelines

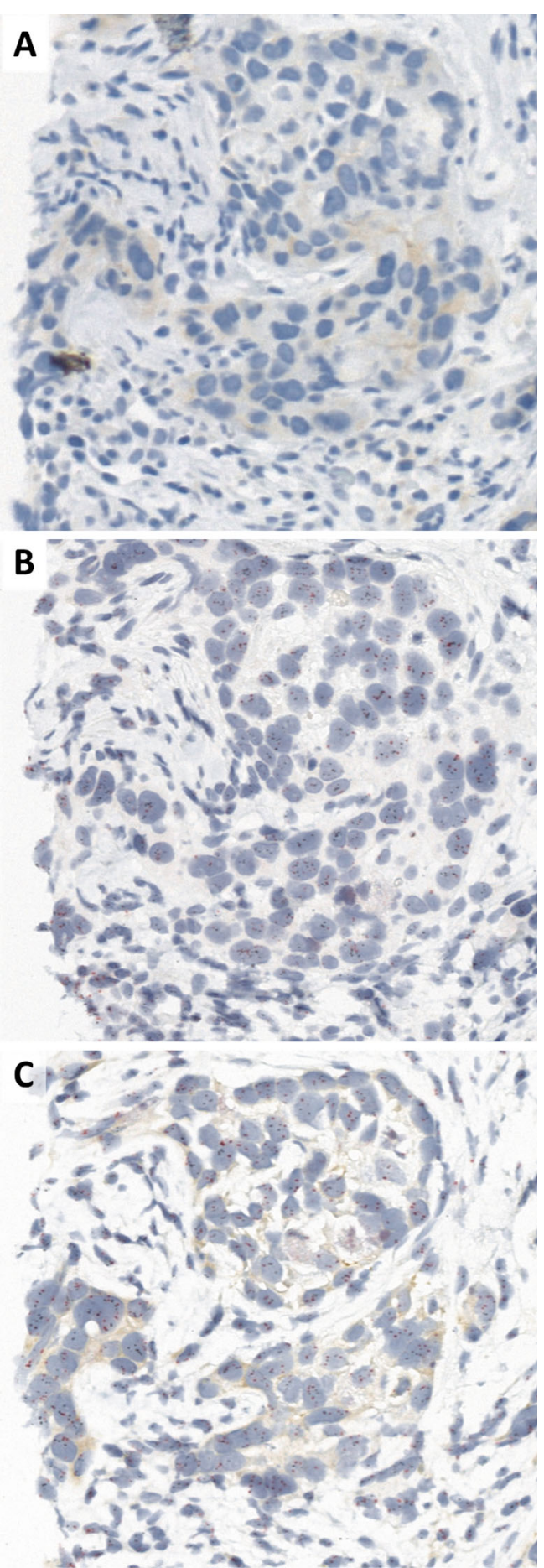

Fig. 2 IHC (a), DISH (b), and GP (c) staining of the same breast carcinoma case at $\times 40$. HER 2 IHC score is determined as 0 for both IHC and GP, with similar staining pattern and intensity (faint cytoplasmic). Low level amplification was detected ( $\geq 6$ copies, ratio above 2 due to minor polysomy) in both DISH and GP slides. This case would not have been eligible for HER2-targeted therapy using IHC alone

or 2 days if the staining is done overnight. Moreover, although the success rate of GP is not perfect, it was better than ISH alone, with only three cases that could not be interpreted after a single staining round. Workflow is also positively affected 
by the use of only a single slide. However, the cost of the GP test is higher than that of the two separate IHC and DISH tests, and therefore, assessment of cost-effectiveness should be conducted to arrive at a comprehensive cost-benefit analysis in terms of treatment optimization [21, 22] .

For borderline cases, we observed that GP reduced the time to get to a final result by $25 \%$. The reading time required for evaluation of 0 and $3+$ cases is not changed in the GP assay, as HER2 protein expression of these cases will be read at low magnification. However, evaluating protein expression and gene amplification in the same slide allows the pathologist to avoid false negative such as in our case with HER2 IHC 0 but HER2 amplification.

The number of tests required to arrive at a final diagnosis increases constantly and will continue to do so in the coming years with new targeted therapies coming along. More tests will require more biological materials, while at the same time clinicians attempt to decrease invasive diagnostic procedures such as surgery or biopsy, which confronts pathologists with reduced sample size. Sample size may become too low to do all required tests with traditional approaches. Consequently, combined testing will be increasingly important to get as much information as possible from little material. GP combines assessment of gene amplification with that of protein expression and provides an elegant option for assessment of HER2 status in breast cancer. As a generic detection method, GP is not limited to one biomarker and provides an opportunity for exploring other marker tests in other diseases.

In conclusion, the combined GP assay improves laboratory workflow and improves patient management by delivering more precise and faster results. A final result for HER2 2+ cases can be delivered within 1 day, while sequential staining requires at least a second day to perform subsequent SISH testing. Efficacy could be further increased by a higher success rate, which needs to be confirmed in further studies.

Acknowledgments This study was partially supported by Roche Tissue Diagnostics France (2 avenue du Vercors, 38240 Meylan) and Ventana Medical Systems Inc. (Tucson, AZ).

Conflict of interest The authors declare that they have no competing interests.

Open Access This article is distributed under the terms of the Creative Commons Attribution 4.0 International License (http:// creativecommons.org/licenses/by/4.0/), which permits unrestricted use, distribution, and reproduction in any medium, provided you give appropriate credit to the original author(s) and the source, provide a link to the Creative Commons license, and indicate if changes were made.

\section{References}

1. Yarden Y, Sliwkowski MX (2001) Untangling the ErbB signalling network. Nat Rev Mol Cell Biol 2(2):127-137
2. Hynes NE (1993) Amplification and overexpression of the erbB-2 gene in human tumors: its involvement in tumor development, significance as a prognostic factor, and potential as a target for cancer therapy. Semin Cancer Biol 4(1):19-26

3. Ménard S, Tagliabue E, Campiglio M, Pupa SM (2000) Role of HER2 gene overexpression in breast carcinoma. J Cell Physiol 182(2):150-162

4. Ross JS, Slodkowska EA, Symmans WF, Pusztai L, Ravdin PM, Hortobagyi GN (2009) The HER-2 receptor and breast cancer: ten years of targeted anti-HER-2 therapy and personalized medicine. Oncologist 14(4):320-368

5. Slamon DJ, Clark GM, Wong SG, Levin WJ, Ullrich A, McGuire WL (1987) Human breast cancer: correlation of relapse and survival with amplification of the HER-2/neu oncogene. Science 235(4785): 182-191

6. Penault-Llorca F, Bilous M, Dowsett (2009) Emerging technologies for assessing HER2 amplification. Am J Clin Pathol 132(4):539-548

7. Bilous M, Morey AL, Armes JE et al (2012) Assessing HER2 amplification in breast cancer: findings from the Australian In Situ Hybridization Program. Breast Cancer Res Treat 134(2):617624

8. Arteaga CL, Sliwkowski MX, Osborne CK, Perez EA, Puglisi F, Gianni L (2012) Treatment of HER2-positive breast cancer: current status and future perspectives. Nat Rev Clin Oncol 9(1):16-32

9. Baselga J, Swain SM (2009) Novel anticancer targets: revisiting ERBB2 and discovering ERBB3. Nat Rev Cancer 9(7):463-475

10. Wolff AC, Hammond MEH, Schwartz JN (2007) American Society of Clinical Oncology/College of American Pathologists guideline recommendations for human epidermal growth factor receptor 2 testing in breast cancer. J Clin Oncol 25(1):118-145

11. Wolff AC, Hammond MEH, Hicks DG et al (2013) Recommendations for human epidermal growth factor receptor 2 testing in breast cancer: American Society of Clinical Oncology/ College of American Pathologists Clinical Practice Guideline Update. J Clin Oncol 31:3997-4013

12. Meijer SL, Wesseling J, Smit VT et al (2011) HER2 gene amplification in patients with breast cancer with equivocal IHC results. J Clin Pathol 64(12):1069-1072

13. Lee JA, Shaheen M, Walke T, Daly M (2011) Clinical and health economic outcomes of alternative HER2 test strategies for guiding adjuvant trastuzumab therapy. Expert Rev Pharmacoecon Outcomes Res 11(3):325-341

14. Bernasconi B, Chiaravalli AM, Finzi G, Milani K, Tibiletti MG (2012) Genetic heterogeneity in HER2 testing may influence therapy eligibility. Breast Cancer Res Treat 133(1):161-168

15. Andersson J, Linderholm B, Bergh J, Elmberger G (2004) HER-2/ neu (c-erbB-2) evaluation in primary breast carcinoma by fluorescent in situ hybridization and immunohistochemistry with special focus on intratumor heterogeneity and comparison of invasive and in situ components. Appl Immunohistochem Mol Morphol 12(1): 14-20

16. Glöckner S, Buurman H, Kleeberger W, Lehmann U, Kreipe H (2002) Marked intratumoral heterogeneity of c-myc and cyclinD1 but not of c-erbB2 amplification in breast cancer. Lab Investig 82(10):1419-1426

17. Seol H, Lee HJ, Choi Y et al (2012) Intratumoral heterogeneity of HER2 gene amplification in breast cancer: its clinicopathological significance. Mod Pathol 25(7):938-948

18. Nitta, H., Kelly, B.D., Padilla, M., et al., A gene-protein assay for human epidermal growth factor receptor 2 (HER2): brightfield tricolor visualization of HER 2 protein, the HER2 gene, and chromosome 17 centromere (CEN17) in formalin-fixed, paraffinembedded breast cancer tissue sections (2012) Diagnostic Pathology, 7 (1), art. no. 60 
19. Hirschmann A, Lamb TA, Marchal G, Padilla M, Diebold J (2012) Simultaneous analysis of HER2 gene and protein on a single slide facilitates HER 2 testing of breast and gastric carcinomas. Am J Clin Pathol 138(6):837-844

20. Osamura RY, Kuwayama A, Katoh M. Simultaneous phenotypic and genotypic heterogeneity at the cellular level approached by HER2 gene-protein assay (GPA) in the invasive ductal carcinoma of the breast: a new approach for cancer pathobiology San Antonio Breast Cancer Symposium 2013-P4-06-12
21. Lidgren M, Wilking N, Jönsson B, Rehnberg C (2008) Cost-effectiveness of HER2 testing and trastuzumab therapy for metastatic breast cancer. Acta Oncol 47(6):1018-1028

22. Garrison LP Jr, Lalla D, Brammer M, Babigumira JB, Wang B, Perez EA (2013) Assessing the potential cost-effectiveness of retesting $\mathrm{IHC} 0$, IHC1+, or FISH-negative early stage breast cancer patients for HER2 status. Cancer 119(17): 3113-3122 